\title{
Mapping the antioxidant activity of apple peels with soft probe scanning electrochemical microscopy
}

\author{
Tzu-En Lin ${ }^{\mathrm{a}}$, Andreas Lesch ${ }^{\mathrm{a}, *}$, Chi-Lin Li ${ }^{\mathrm{b}}$, Hubert H. Girault ${ }^{\mathrm{a}, *}$ \\ a École Polytechnique Fédérale de Lausanne, EPFL Valais Wallis, Laboratoire d'Electrochimie Physique et Analytique, rue de l'Industrie 17, Case Postale 440, CH-1951 Sion, Switzerland \\ b Department of Chemistry, National Taiwan University, 1, Section 4, Roosevelt Road, Taipei 10617, Taiwan
}

\section{A R T I C L E I N F O}

\section{Article history:}

Received 26 September 2016

Received in revised form 3 January 2017

Accepted 4 January 2017

Available online 06 January 2017

\section{Keywords:}

Scanning electrochemical microscopy

Soft probes

Microelectrodes

Antioxidants

Apple peel

\begin{abstract}
A B S T R A C T
We present a non-invasive electrochemical strategy for mapping the antioxidant (AO) activity of apple peels, which counterbalances oxidative stress caused by various external effectors. Soft carbon microelectrodes were used for soft probe scanning electrochemical microscopy (SECM) enabling the gentle and scratch-free contact mode scanning of rough and delicate apple peels in an electrolyte solution. The SECM feedback mode was applied using ferrocene methanol $(\mathrm{FcMeOH})$ as redox mediator that gets electrochemically oxidized at the soft probe and diffuses towards the apple peel where it gets regenerated by certain AOs leading to a redox mediator recycling and increased current signal. The global AO activity in the apple peel including lenticels and regions with artificially degraded AOs were mapped using the soft microelectrodes. Finally, in an apple cross-section the higher and homogeneous AO concentration in the peel with a heterogeneously decaying AO gradient towards the apple inward was visualized, demonstrating the adequate micrometer resolution of the SECM probe and the possibility to get information of the interior AO activity of the apple.
\end{abstract}

(C) 2017 Elsevier B.V. All rights reserved.

\section{Introduction}

Antioxidants (AOs) are well-known beneficial dietaries for reducing the risks of cardiovascular disease and cancer [1-3]. They also protect fruits from oxidative stress caused by unfavorable external environments such as drought, chilling injury, UV radiation or pathogen attack [4-7]. Hence, AOs in fruits are generally more concentrated in the peel than in the flesh representing a major fruit protection barrier $[8,9]$. Several enzymatically assisted mechanisms provide a certain degree of regeneration of AOs after the AOs degradation by free radicals [10]. Consequently, the $\mathrm{AO}$ concentration in the peel is a quantitative indicator for the status of the AO defense system of the fruit.

Apples have a high content of AOs making them highly valuable crops from which thousands of tons are consumed worldwide every day. Therefore, the understanding and support of their AO defense system is an important issue for apple cultivators [11,12]. The AO defense system of apples is rather complex and depends on many factors, such as apple variety and growing as well as storage conditions [13]. It has been reported that polyphenolic compounds, in particular flavanols (e.g., catechin and procyanidin), flavonoids (e.g., quercetin) and hydroxycinnamic acid derivates (e.g., chlorogenic acid), show the highest $\mathrm{AO}$ activity $[6,7,14]$. Other $\mathrm{AO}$ molecules, such as glutathione

\footnotetext{
* Corresponding authors.

E-mail addresses: andreas.lesch@epfl.ch (A. Lesch), hubert.girault@epfl.ch (H.H. Girault)
}

(GSH) or ascorbate (vitamin C, L-AA), participate in the removal of free radicals [15]. Although L-AA is up to six times more concentrated in the apple peel than in the fruit part, the contribution of L-AA to the overall AO activity is rather small [11]. Antioxidative enzymes, such as glutathione reductase, ascorbate peroxidase and catalase, are involved in reducing free radicals and $\mathrm{H}_{2} \mathrm{O}_{2}$, while they also keep the level of GSH and L-AA at sufficient levels within the glutathione-ascorbate cycle [10]. The major part of these AOs is fixed in the apple matrix as glycosides and carboxylic acid esters [16,17], which usually requires vigorous extraction and purification methods such as HPLC followed by the quantification with optical detection methods $[18,19]$. The sample preparation and optical measurement procedures are time consuming and optical methods can be interfered by sample color background (color pigments in fruits) or auto-fluorescence [20-22]. Furthermore, although the peel and flesh of the apple can be separated prior to the extraction and $\mathrm{AO}$ quantification, spatial information about the local distribution of AOs, e.g., over the peel surface, cannot be obtained. In addition, once an apple is prepared for analysis using the invasive methods described above, the AO content of this particular fruit cannot be followed further requiring the investigation of statistically relevant amounts of apples for aging studies.

Therefore, developing simple and non-destructive sensors for analyzing rapidly, at low cost and on site the status of the AO defense system with high spatial resolution is attractive for apple growers. UV sensitive AOs in the outer layer of the apple peel have recently been measured directly and non-invasively on the fruit by using reflectance 
[23] and chlorophyll fluorescence spectroscopy [24,25]. However, these approaches can only be applied to optically active compounds. Alternatively, AOs can be detected at amperometric electrodes where the AOs get electrochemically oxidized or reduced depending on the applied electrode potential [26]. The recorded current is proportional to the concentration of AOs in the sample and common optical limitations (e.g. interferences from colorful pigments) do not occur. For this analysis, the AOs need to be extracted from the apple and dissolved in an electrolyte solution. Nevertheless, the electrochemical detection of certain AOs can suffer from slow kinetics and/or interferences by other electrochemically active compounds. One approach is to use redox mediators that, for instance, get reduced by the AOs and then electrochemically oxidized at the electrode resulting in a redox mediator recycling [27]. The standard redox potential of the AOs must be lower than the one of the redox mediator. These redox active compounds are generally embedded in the matrix of chemically modified electrodes [28] but can also be present in solution $[29,30]$. The latter concept is related to the wellknown ferric ion reducing antioxidant power (FRAP) assay where $\mathrm{Fe}^{3+}$ ions are reduced by AOs to $\mathrm{Fe}^{2+}$ ions to determine the total AO's reducing capacity by a detectable color change of the iron complex [5, 31].

Micrometer spatial resolution with electrochemical methods can be achieved by using scanning electrochemical microscopy (SECM) where microelectrodes are laterally translated in close vicinity to a substrate inside an electrolyte solution containing redox active species [32-38]. Typical SECM applications range from bio-imaging, surface reactivity mapping to the characterization of liquid-liquid interfaces [39-42]. Conventionally, SECM is operated in constant height mode requiring flat and non-tilted substrates. Natural samples such as apples contain curved topographic features that are reasonably larger than the probe dimension. This leads to misleading information caused by the varying working distance between the probe and the sample. Moreover, the microelectrode and substrate could physically contact leading potentially to microelectrode contamination as well as sample/probe damages. Several constant distance SECM approaches have been reported, mainly by the combination of SECM with scanning probe techniques that include a constant distance option [43-48]. However, this requires advanced instrumentation and probe manufacturing [49-51]. The common goal of most constant distance SECM approaches and related techniques is the improvement of the imaging resolution to the nanoscale, mainly by reducing the probe dimensions [49,52-54]. However, the typical micrometer resolution of standard SECM probes is still sufficient for many biological samples, such as tissues, where the analysis of relatively large areas exceeding square millimeters is required [55].

In this work, we employed soft SECM probes that are made of thin and flexible polymeric materials with an implemented carbon microelectrode enabling gentle contact mode scanning over delicate samples, i.e., soft probe scanning electrochemical microscopy $[55,56]$. Such scanners are ideal for measurements on the fragile and curved peel of apples (Scheme 1). Ferrocene methanol (FcMeOH) was used as redox mediator that was electrochemically oxidized under diffusion control to $\mathrm{FcMeOH}^{+}$at the scanning microelectrode. The result is a steady-state current. When the probe is brought in close proximity to the sample, $\mathrm{FcMeOH}^{+}$reaches by diffusion the apple peel where the reaction of $\mathrm{FcMeOH}^{+}$with the AOs inside the apple peel regenerates $\mathrm{FcMeOH}$ (known as SECM feedback mode). This additional flux of FcMeOH towards the microelectrode results in an increased current signal (i.e., "regeneration current") compared to a lower current over purely insulating substrates where no regeneration takes place and just hindered diffusion of the redox mediator is observed. In this way, the AO activity initiated by molecular and enzymatic systems in the apple peel and able to reduce $\mathrm{FcMeOH}^{+}$was mapped. Similarly, $\mathrm{FcMeOH}$ had been used previously by other groups in SECM related approaches to detect the status of the AO molecule GSH in cancer cells [57,58]. Furthermore, an apple peel sample was exposed to hydrogen peroxide solution to degrade artificially the AO activity as clearly visualized by

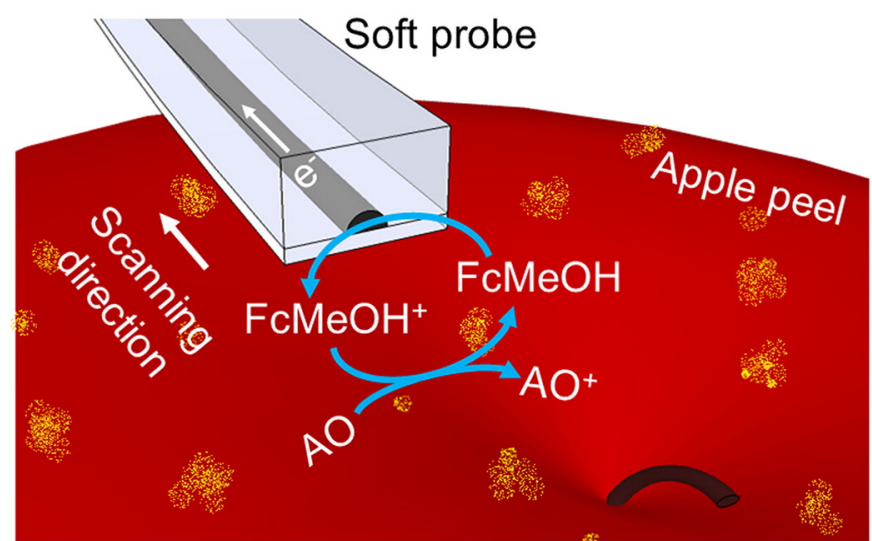

Scheme 1. Schematic representation of a soft probe detecting AOs on a curved apple surface by the regeneration current of $\mathrm{FCMeOH}$ caused by AOs present in the apple peel.

subsequent SECM imaging. Finally, the AO activity and the gradient of AO concentration in an apple cross-section were mapped.

\section{Experimental section}

\subsection{Chemicals}

FcMeOH, monopotassium dihydrogen phosphate, dipotassium monohydrogen phosphate (all from Sigma-Aldrich) and hydrogen peroxide (60\%, Fisher Chemicals) were of analytical grade and used as received. Deionized water was produced by a Milli-Q plus 185 model (Millipore). Gala apples were purchased from a local market. Polyvinylidene fluoride (PVDF) membrane was bought from Bio-Rad (Switzerland).

\subsection{Fruit sample preparation}

A thin slide of apple peel was cut out from the apple with a peeler, placed on a glass slide and cleaned with isopropanol and a soft wipe to remove possible protective layers and other contaminants from previous agricultural, transport and storage treatments. Most importantly, this procedure removed also the highly hydrophobic natural wax layer on the apple peel to allow the permeation of the electrolyte solution into the apple peel. In general, the apple skin consists of a natural epicuticular wax layer (sub- to several micrometer thickness) covering the cuticle (22-55 $\mu \mathrm{m}$ ), epidermis and hypodermis [59]. The natural wax layer is water repellent and reduces the water as well as hydrophilic AO permeability through the apple skin, but can carefully be removed with organic solvents $[60,61]$. Using rapidly and gently an isopropanol wetted wipe instead of immersing the apple peel into isopropanol should preserve most of the AO activity. Previous works have demonstrated that the alcohol based removal of the cuticular wax layer on apples was performed without damaging the epidermal cells [61]. However, before immersion the apple slide was quickly covered with a rectangular frame made of Parafilm to expose a fruit peel area of approximately $0.5 \mathrm{~cm}^{2}$. The electrochemical cell for SECM experiments was completed by a cylindrical Teflon cover mounted on the top and filled with the electrolyte solution (SI-1).

\subsection{Micro-contact printed apple cross-section on PVDF membrane}

The apple cross-section was cut from a cleaned apple with a scalpel blade. A PVDF membrane was wetted in methanol for $5 \mathrm{~s}$ and washed in water for $2 \mathrm{~min}$. The apple cross-section was put in contact with the PVDF membrane for 15 min before the PVDF membrane was placed after a membrane drying period into the electrochemical cell for SECM experiments. 


\subsection{Soft probes for Soft-Probe-SECM}

Soft carbon microelectrode probes (VersaScan (VS) Stylus probes obtained from Princeton Applied Research - Ametek) were prepared according to a fabrication procedure previously introduced and established by our group (SI-2) [62,63]. Briefly, the soft probes consist of $100 \mu \mathrm{m}$ thick polyethylene terephthalate sheets with microchannels of $25 \mu \mathrm{m}$ width and $15 \mu \mathrm{m}$ depth filled with a carbon paste and coated with a $2 \mu \mathrm{m}$ thick Parylene C layer. A soft probe was then cut into a Vshape by using a razor blade to form a tiny tip slightly larger than the width of the active carbon microelectrode area, which itself had been exposed by another sharp razor blade cut (SI-2).

\subsection{SECM measurements}

A customized SECM setup running under SECMx software (University of Oldenburg, Germany) [64] comprising a CompactStat (Ivium Technologies), and an electronic sample tilt table (Zaber Technologies) was used. The soft probes were used as working electrodes while a threeelectrode arrangement was completed by using a $\mathrm{Ag}$ wire as quasi-reference electrode and a platinum wire as counter electrode. SECM data plotting was carried out using MIRA software (University of Oldenburg, Germany). All experiments were performed at room temperature $\left(20^{\circ} \mathrm{C}\right)$. The probe was fixed in a custom-made holder with an inclination angle of $20^{\circ}$ between the probe and the surface normal of the substrate for pre-setting the bending direction of the probe and ensuring a good current contrast during SECM imaging in contact mode. The mechanical probe-substrate contact was quantified using a quantity $h_{\mathrm{p}}$. This probe height represents the theoretical vertical probe displacement after the mechanical contact with the substrate assuming the probe would penetrate into the sample. This $h_{\mathrm{P}}$ is zero when the probe contacts the substrate and becomes negative when the probe starts to slide on the sample surface as a result of the continued downwards movement of the probe holder. In this work, the $h_{\mathrm{P}}$ value was set to $200 \mu \mathrm{m}$ to ensure a permanent physical contact of the soft probe and the apple peel during the experiments. During SECM imaging, a lift-off routine was applied to retract the probe into the bulk solution during reverse scanning in order to avoid probe over-bending and strong mechanical forces to the probe as well as to the sample [56]. In all SECM imaging experiments, the following further parameters were used: working electrode potential $E_{\mathrm{T}}=0.4 \mathrm{~V}$, probe translation speed $=$ $50 \mu \mathrm{m} / \mathrm{s}$, step size $=25 \mu \mathrm{m}$ and delay time between probe movement

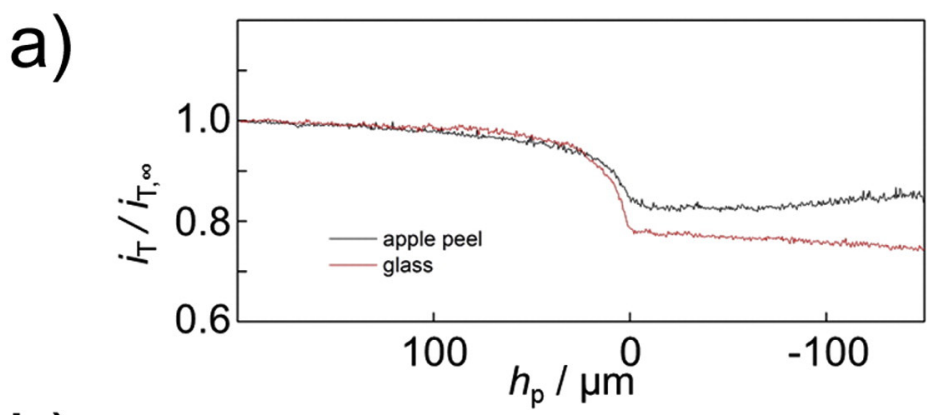

b)

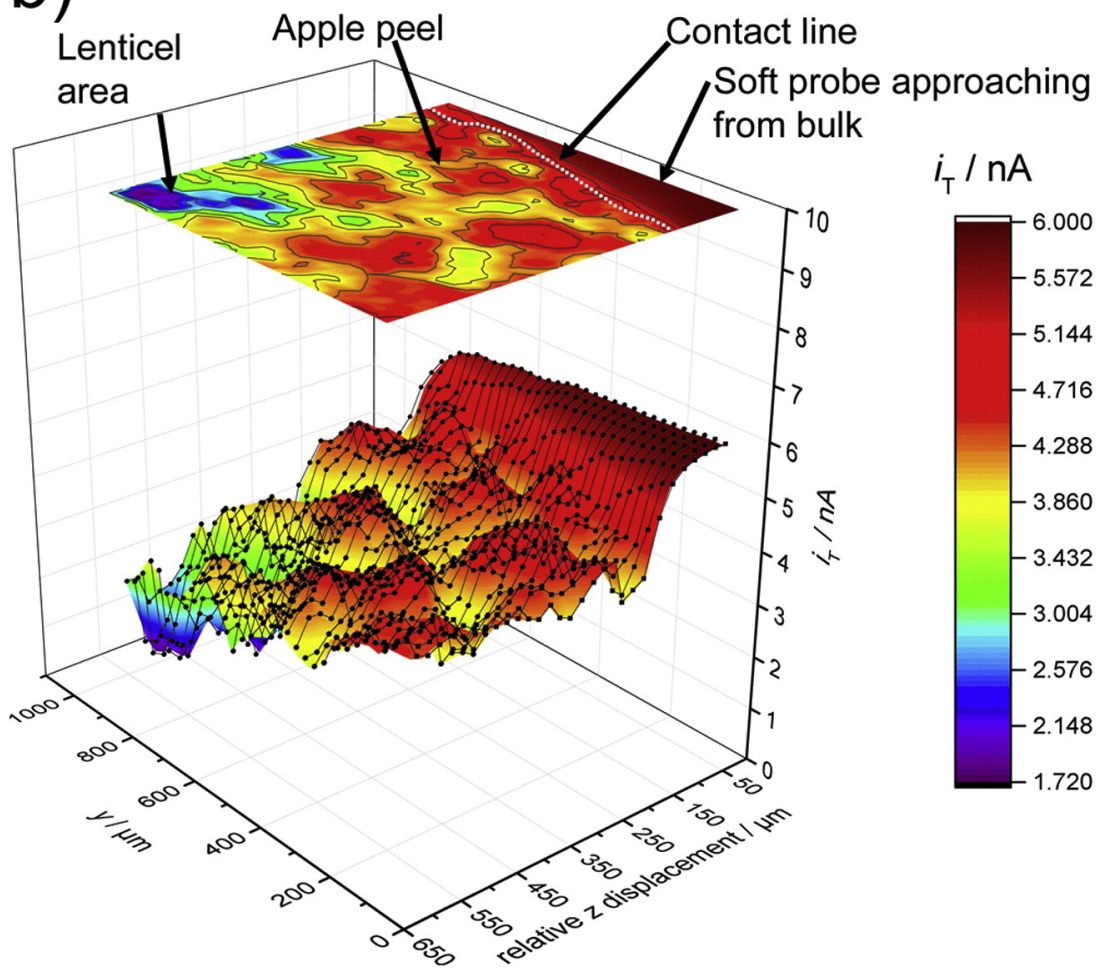

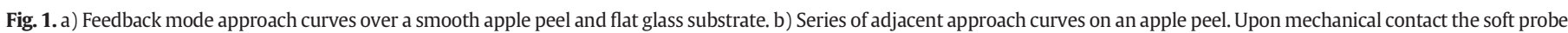

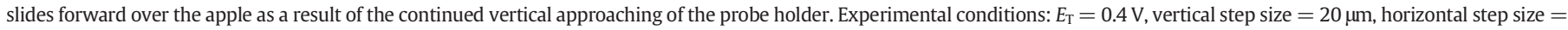

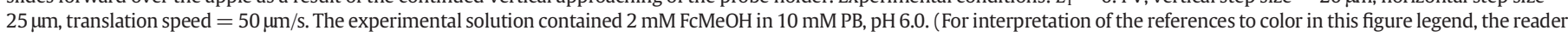
is referred to the web version of this article.) 
and current reading $=0.1 \mathrm{~s}$. The experimental solution contained $2 \mathrm{mM}$ $\mathrm{FcMeOH}$ in $10 \mathrm{mM}$ phosphate buffer (PB), pH 6.

\section{Results and discussion}

When the carbon microelectrode is positioned in the bulk solution (i.e., $h_{\mathrm{P}} \gg 0$ ), a diffusion-limited steady-state current $i_{\mathrm{T}, \infty}$ for the electrochemical oxidation of $\mathrm{FcMeOH}$ is recorded. While the probe is approaching an insulating glass surface the diffusion of $\mathrm{FcMeOH}$ gets physically hindered by the glass substrate and the recorded tip current $i_{\mathrm{T}}$ decreases (red curve in Fig. 1a with normalized current $i_{\mathrm{T}} / i_{\mathrm{T}, \infty}$ ) until the soft probe touches the glass surface $\left(h_{\mathrm{P}}=0\right)$. At this point the soft probe starts sliding forward over the glass surface while the working distance $d$ is kept constant resulting in a barely changing current. The remaining current is a result of the diffusion of $\mathrm{FcMeOH}$ from the bulk solution around the soft probe tip. When approaching a smooth part of an apple peel with the same soft probe and within the same electrochemical cell (black curve in Fig. 1a), the measured tip current decreases less strongly and is larger at physical contact with the apple peel compared to the theoretical negative feedback current for a totally inactive substrate such as glass. This is an indication for redox mediator regeneration induced by the apple peel AOs. Indeed, the recorded regeneration current is smaller than the diffusion-controlled oxidation current of $\mathrm{FcMeOH}$ in bulk solution demonstrating finite kinetics of the
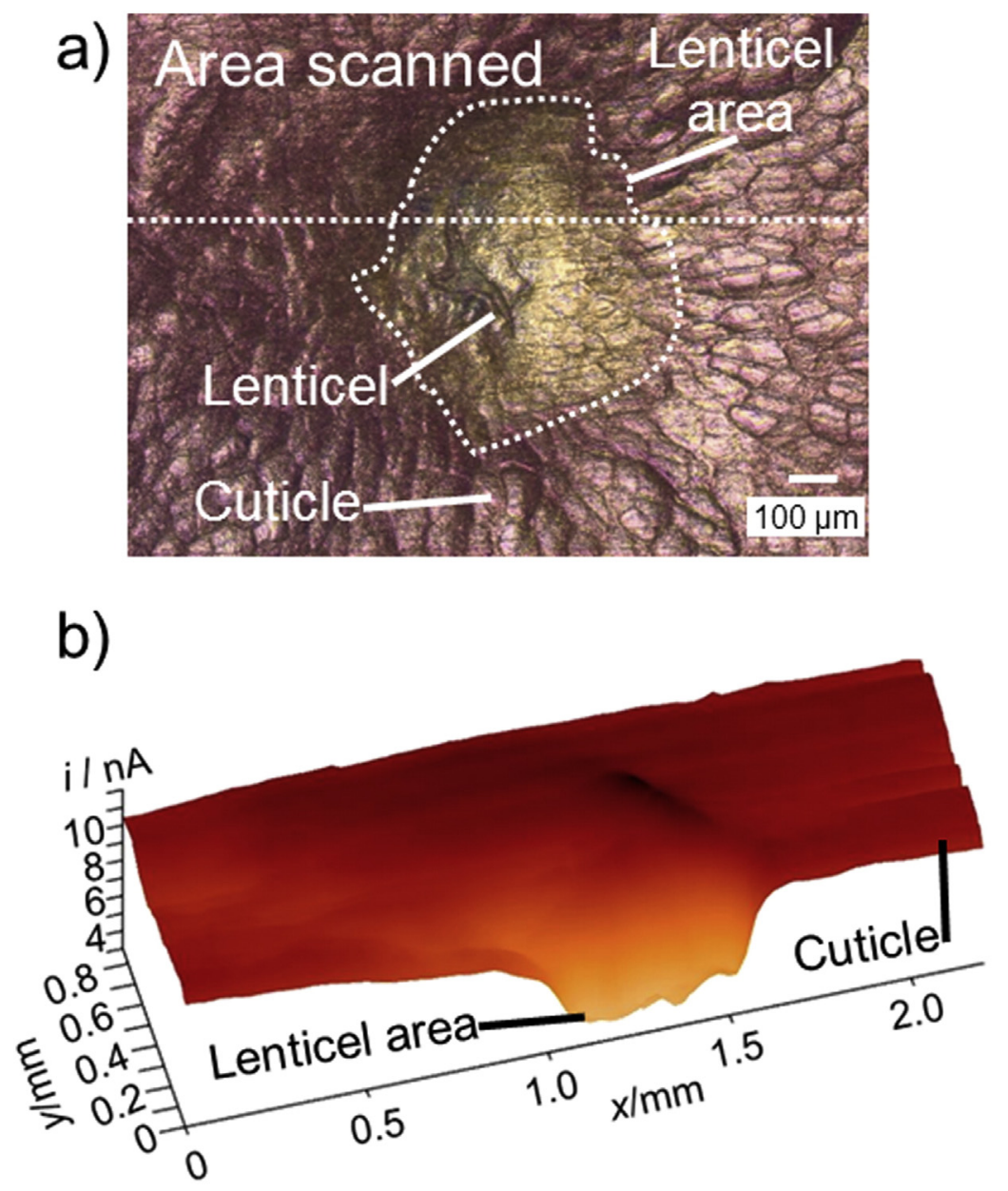

c)

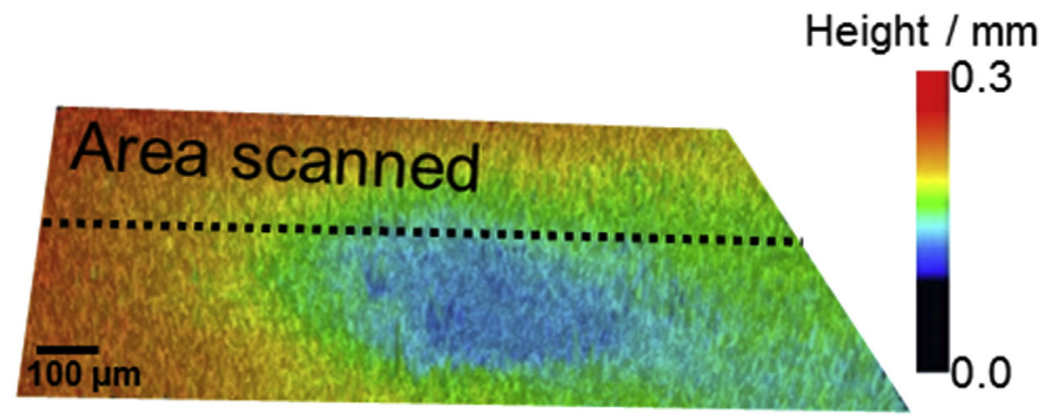

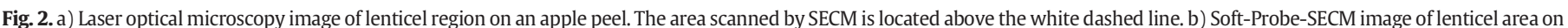

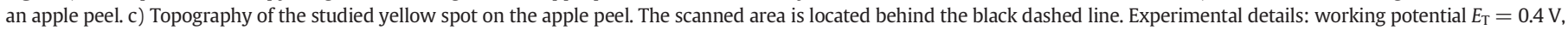

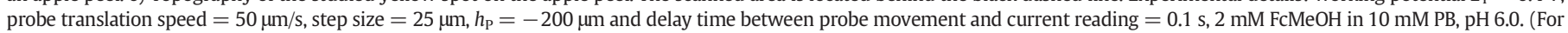
interpretation of the references to color in this figure legend, the reader is referred to the web version of this article.) 
a)

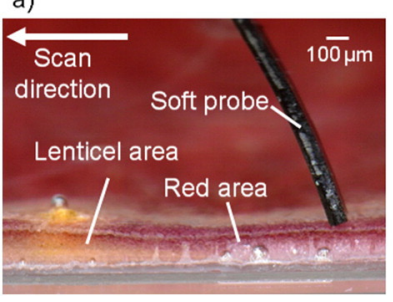

b)

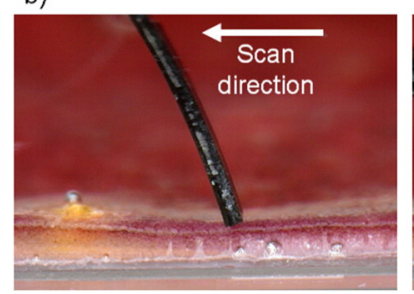

c)

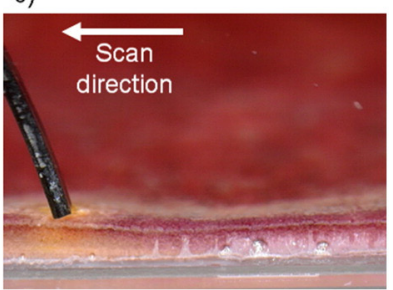

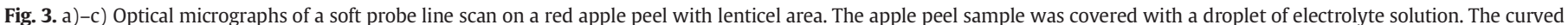

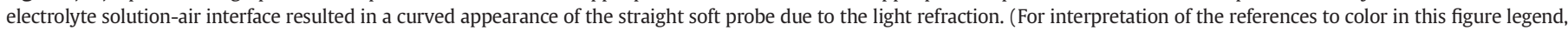
the reader is referred to the web version of this article.)

regeneration reaction, a small amount of accessible active AO centers and/or a slow mass transport of $\mathrm{FcMeOH}$ through the various layers of the apple peel matrix with likely different porosity. Besides these three effects, the microscopic apple peel topography could influence the measured current signal by influencing the diffusion of the redox mediator (SI-3). A varying sample tilt shows a limited influence on the recorded current as demonstrated previously [65]. Furthermore, organic compounds, which are electrochemically inactive in the applied electrochemical window, could slowly diffuse out from the apple peel and contaminate the electrode surface. Consequently, the absolute current measured at the electrode goes down (vide infra), whereas electrode fouling was not observed when measuring the glass sample.

Generally, the reaction of free radicals with AOs is very fast. However, most AOs are embedded inside the apple peel and the exact transport mechanism of $\mathrm{FcMeOH}$ into the apple peel compartments is unknown yet. It is known that an intracuticular lipophilic wax is present within a more hydrophilic cutin polymer matrix in the cuticular layer of the apple peel forming a water-in-oil emulsion like structure [61]. This layer contains certain amounts of the hydrophilic and lipophilic AOs (vide supra) [61]. Because FcMeOH shows neither unequivocal hydrophilic nor hydrophobic characteristics, it was chosen as the redox mediator in this study. Furthermore, SECM approach curves, such as the ones shown over the glass and the apple peel, do theoretically not cross each other, because the shape of such curves is mainly dependent on the substrate kinetics. The observed crossing in Fig. 1a is most likely a result of the macroscopic apple topography that affects the diffusion of $\mathrm{FcMeOH}$ and influences the tip current as long as the soft probe is not in contact with the substrate.

The validity of the feedback mode concept was supported by cyclic voltammetry in solutions containing various concentrations of the AOs quercetin and GSH, which caused a clear amplification of the steadystate current for the oxidation of FcMeOH (SI-4) [66]. In order to demonstrate the stability and reproducibility of approach curves along such rough and delicate surfaces, a series of 31 parallel approach curves on the apple peel was performed and the recorded tip current was plotted as a function of the horizontal and vertical probe position (Fig. 1b). Once the soft probe got in contact with the sample and started a horizontal probe movement on the apple peel, the recorded approach curve experiments turned into a 2D mapping of the substrate reactivity. Such combined approach curve-imaging experiment demonstrated the stability of the probe sliding as well as a stable electrochemical signal. While sliding over the apple peel surface, regions of higher (yellowred in Fig. 1b) and lower (blue) redox mediator regeneration capabilities were identified.

The origin of these signals is a non-uniform apple peel surface that contains specific microstructures of which some appear for instance as yellow spots (Fig. 2a). These areas consist of special cells called lenticels (SI-5), which are porous tissues with large intercellular spaces located in the periderm of fruit peels. The lenticels are formed from cracks that arise when the fruit peel expands during apple growing and can act as pores for the interchange of gases between the interior tissue and the surrounding air. The AO defense system of a fruit starts a mechanism to accumulate AOs, mainly phenolic compounds, around such lenticel to protect the fruit from oxidative stress induced by the air entering through this porous structure $[67,68]$. As a result of this defense mechanism, the phenolic AOs located around a lenticel are mainly oxidized leading to lenticel discoloration. This can be seen as the yellowish area surrounding the lenticel, termed lenticel area in this work (Fig. 2a).

A soft probe was then applied to monitor the AO activity around a lenticel on the apple peel by using the lateral SECM imaging routine where the probe was horizontally translated in a line-by-line fashion (Fig. 2b). The current over the lenticel and in the surrounding area was significantly lower than over the surrounding apple peel indicating the lower amount or absence of AO activity in this so-called lenticel area (i.e. the lenticel with periphery of reduced AO activity). It is important to note that this yellow spot area had a maximum lateral dimension of about $0.8 \mathrm{~mm}$ with a maximum depth of $100 \mu \mathrm{m}$ (Fig. 2c). Due to the contact mode operation, the tip of the soft probe was able to slide inside this lenticel area that was larger than the probe width (i.e., $100 \mu \mathrm{m}$ ) and that showed a flat slope towards the center of the lenticel area providing a constant working distance. For instance, if the probe width would be much larger than the diameter of the yellow spot and the sample aspect ratio would be high the probe would not enter inside this lenticel area. Consequently, $d$ would increase and the result would be a larger current approaching the steady-state current in bulk solution over the $800 \mu \mathrm{m}$ wide lenticel area, but the opposite was the case (Fig. 2b). However, the situation is different for the lenticel itself. The tip of the soft probe is generally wider than the width of typical lenticel cracks. If such cracks have significant depths, topographic effects in the current measurement are certainly possible assuming the lateral imaging resolution as achieved by the probe dimension and the experimental conditions is high enough. No significant topography of the lenticel crack was

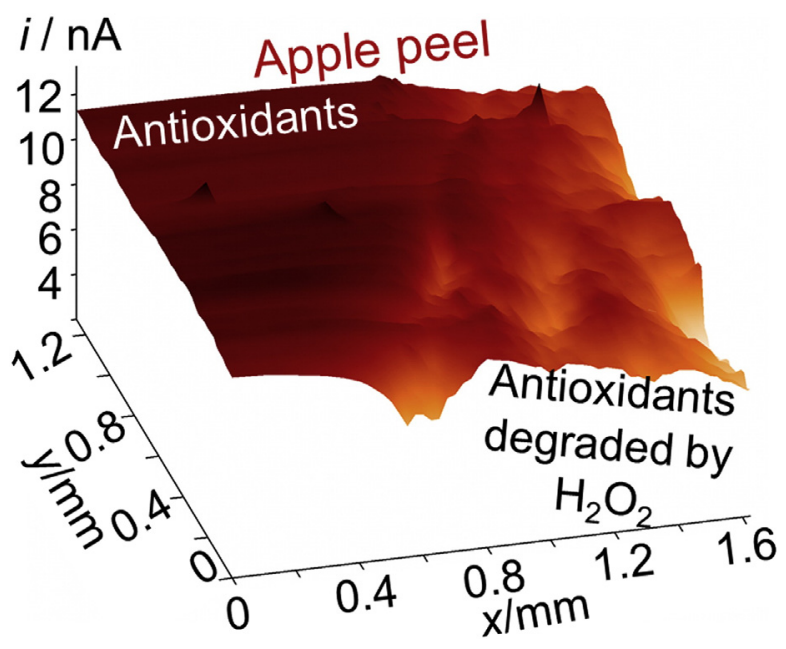

Fig. 4. Soft-Probe-SECM image of an apple peel area partially exposed to $\mathrm{H}_{2} \mathrm{O}_{2}$ for 15 min prior to the experiment. Experimental details: working potential $E_{\mathrm{T}}=0.4 \mathrm{~V}$, probe translation speed $=50 \mu \mathrm{m} / \mathrm{s}$, step size $=25 \mu \mathrm{m}, h_{\mathrm{P}}=-200 \mu \mathrm{m}$ and delay time between probe movement and current reading $=0.1 \mathrm{~s}, 2 \mathrm{mM} \mathrm{FcMeOH}$ in $10 \mathrm{mM}$ PB, pH 6.0. 
detected in the laser scanning microscope image (Fig. 2c). It is worth noting, that lenticels can show different geometry and topography depending on their type, developmental stage and apple variety.

The redox mediator regeneration is most likely caused by the AOs and related AO enzymatic mechanisms, which could be i) embedded inside the cuticle (redox mediator would diffuse in and out the cuticle), ii) fixed in the top layer of the cuticle (easily accessible for the redox mediator) or iii) diffusing out from the cuticle (regeneration of the redox mediator by AO molecules or direct measurement of AOs outside from the apple). The SECM imaging of the apple peel in phosphate buffer solution without redox mediator has not shown any significant current signal (SI-6). This explains that the direct electrochemical oxidation of AOs

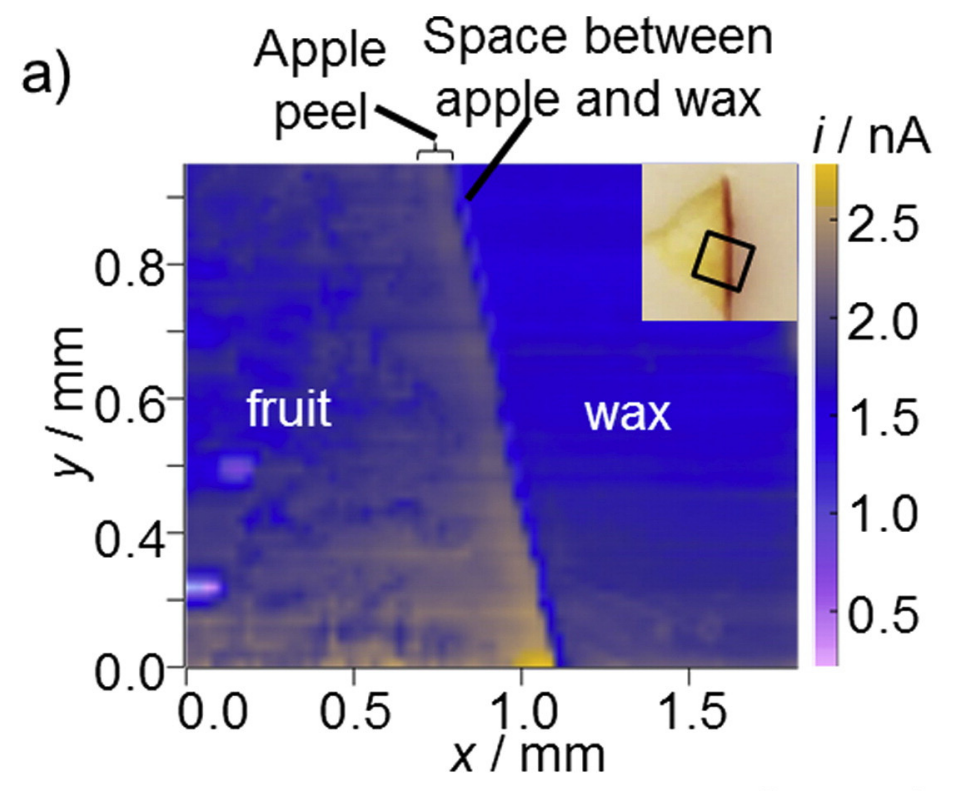

b)
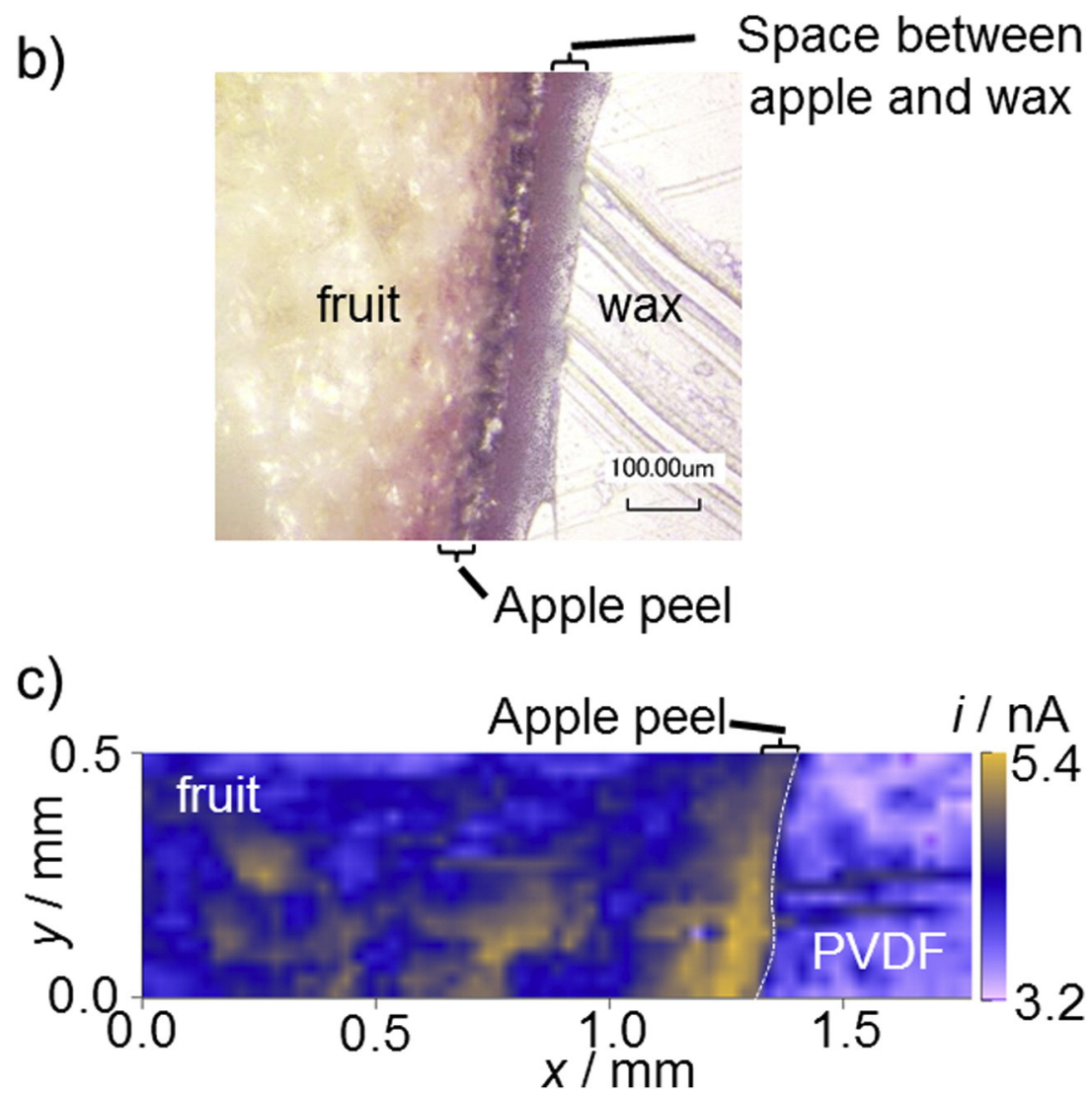

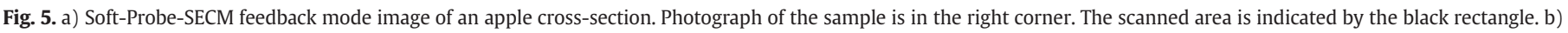

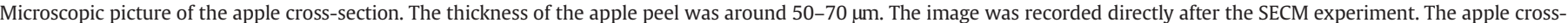

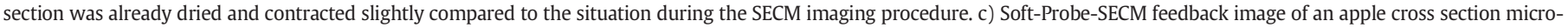

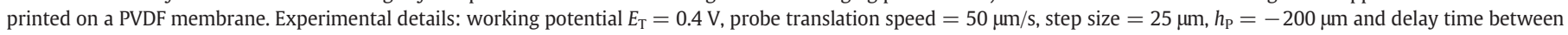
probe movement and current reading $=0.1 \mathrm{~s}, 2 \mathrm{mM} \mathrm{FcMeOH}$ in $10 \mathrm{mM} \mathrm{PB}$, pH 6.0. 
released from the apple can be excluded, but the amplification by $\mathrm{FcMeOH}$ recycling is still possible. The exact underlying processes are not fully understood yet. SECM experiments in pure buffer solution after a prior immersion of FCMeOH in the SECM imaging solution with subsequent apple peel washing step have shown that a significant amount of $\mathrm{FcMeOH}$ enters and accumulates in the apple peel due to the peel's permeability and possibly porosity (SI-7). Although red apple peel and lenticel area showed a comparable accumulation, there might still be a different permeability for the redox mediator affecting the reported results. This still needs to be confirmed in the future, but it can be suggested that the present feedback mode approach recognizes in part the global AO activity that is able to reduce $\mathrm{FcMeOH}^{+}$.

The pressure that is exerted by the soft probe onto the substrate is rather small as previously demonstrated by melanoma tissues and self-assembled monolayers that were brushed without detectable damage $[55,56]$. In this work, we have recorded a video sequence of a soft probe sliding along the vertical cross-section of an apple peel, including a lenticel area, in order to identify a possible deformation and compression of the apple peel (SI-8). In Fig. 3, three representative snapshots from the video are shown. The soft probe followed the topography of the apple peel and lenticel area while the peel surface seemed to get neither deformed nor compressed. However, when approaching the apple peel surface area, the soft probe slit forwards and the apple peel was slightly compressed (SI-8). This might cause the release of (electro)chemically active compounds, however, no indications for this were observed in the experiments performed in this work (for instance, see approach curve plot in Fig. 1b).

In order to corroborate that the distribution of the AO activity within the apple peel can be visualized with high certainty, part of the apple peel components was chemically oxidized by a controlled exposure to hydrogen peroxide $\left(\mathrm{H}_{2} \mathrm{O}_{2}\right)$ solution for 15 min prior to SECM imaging. Such treatment led to a strong artificial oxidative damage due to the oxidizing power of $\mathrm{H}_{2} \mathrm{O}_{2}$ for biological materials, which is directly linked to a significant degradation of the AO defense system. This might be accompanied by a change in the permeability characteristics of this peel area. The boundary between the untreated and treated areas is clearly visible in the SECM feedback mode image (Fig. 4). A lower current was measured over the bleached area probably due to the reduced AO activity. Some of the present AOs, such as anthocyanidin, are also pigments that change color or turn even colorless after oxidation (SI-9). However, many AOs, such as GSH, are colorless and their degradation cannot be identified simply by eye or other optical methods, but by using the presented SECM imaging strategy.

Furthermore, the higher content of AOs in the apple peel was confirmed by SECM feedback mode imaging of the cross-section of an apple (Fig. 5a). After the cutting procedure, the sample was quickly embedded in a flat wax matrix and immersed in solution. Over the wax area, a pure hindered diffusion current was measured (schematic representation of the soft probe movement over the wax-apple peal boarder in SI-10). Over the apple peel, the small current gradient from higher values (lower part of the SECM image) to lower values (upper part of the SECM image) was due to slow and slight electrode fouling, most likely caused by compounds diffusing slowly out of the apple and contaminating the carbon electrode surface. The apple peel of approximately $100 \mu \mathrm{m}$ thickness (from right to left containing the cuticular, epidermal and hypodermal layers) shows a higher and homogeneous AO activity in respect to the flesh (Fig. 5a), which is also valid when considering the decaying electrode current and comparing the currents for each $y$-position. On the contrary, a locally heterogeneous gradient of AO activity from the peel towards the flesh can be seen. The photograph of the apple cross-section after the SECM experiment is shown in Fig. 5b. Please note, that the gap between apple peel and wax in the optical image is larger than it was during the actual experiment due to the water loss in the apple matrix while drying at air. This experiment demonstrates that Soft-Probe-SECM imaging can be used to investigate the distribution of the AO activity in various parts of the apple, even in the fruit part. Imaging on the flesh is more challenging since various biological compounds can diffuse in the electrolyte solution causing serious electrode contamination issues over time (seen as a minor current decrease in positive $y$-direction). In order to show that the SECM image of the apple cross-section represents the global AO activity a freshly prepared apple cross-section was micro-contact printed onto a PVDF membrane to adsorb the hydrophobic AOs molecules and proteins (Fig. 5c). PVDF membranes are well known for their extraordinary ability to adsorb proteins and hydrophobic molecules and have previously been used by our group for detecting tyrosinase enzymatic activity in bananas [42]. The left image part (i.e. $x=0-1.2 \mathrm{~mm}$ ) corresponds to the apple fruit, whereas the area between $x=1.2-1.3 \mathrm{~mm}$ shows the enhanced AO activity of the apple peel. This result is in very good agreement with the SECM image performed directly on the apple cross-section. However, the permeability for the redox mediator is expected to be higher in the exposed apple peel cross-section and flesh compared to the top of the peel. Because the AO activity of the peel and flesh was preserved and gave the same result in the printed sample it can be suggested that the permeability of the redox mediator over the entire sample is comparable and/or does not influence this measurement.

\section{Conclusions}

The local distribution of antioxidant activity on apple peels and within apple peel cross-sections was efficiently mapped using SoftProbe-SECM, which has recently been developed and intensively used for corrugated biological surfaces. Thanks to the weak forces exerted onto the sample surface and due to the bending properties of the soft probes a constant working distance was easily achieved by contact mode scanning without inducing damages, neither to the apple substrate nor to the probes. The SECM feedback mode was successfully applied to record an enhanced current over AO-rich locations due to the redox mediator regeneration of $\mathrm{FCMeOH}$ initiated by the AO defense system of the apple peel and flesh. In this way, the AO activity on red apple peel cuticle (high), on the yellow lenticel areas (lower) and on areas with artificially degraded AO activity (very low) has been visualized. Furthermore, the higher and homogeneous AO activity in the peel compared to the flesh was confirmed. However, the possible influence of the peel permeability for the redox mediator, which is part of further ongoing research, needs to be considered for the interpretation of the presented data.

The presented electrochemical approach could be the basis for further research and development of electrochemically based in-field antioxidant sensors and scanners. Non-invasive monitoring on apple peels of intact apples could be achieved by implementing the soft microelectrode into portable, handhold scanners for in-field measurements. The electrolyte solutions could be inserted into a hydrogel or delivered and aspirated from microfluidic channels $[69,70]$. Such scanners would be attractive for the monitoring of the antioxidant defense of apple peels during growing, picking and storing, for the early identification of fruit diseases, such as lenticel breakdown.

\section{Acknowledgements}

T.-E. L. thanks the Ministry of Education (MOE), Taiwan, for the 2013 MOE Technologies Incubation Scholarship. A. L. thanks the Swiss National Science Foundation (SNSF, project No. 154297). The collaboration with Princeton Applied Research-Ametek for the soft probe distribution is acknowledged. Dr. Fernando Cortés-Salazar (EPFL) and Prof. Gunther Wittstock (University of Oldenburg, Germany) are thanked for their academic assistance.

\section{Appendix A. Supplementary data}

Supplementary data to this article can be found online at http://dx. doi.org/10.1016/j.jelechem.2017.01.015. 


\section{References}

[1] H.N. Siti, Y. Kamisah, J. Kamsiah, The role of oxidative stress, antioxidants and vascular inflammation in cardiovascular disease (a review), Vasc. Pharmacol. 71 (2015) 40-56.

[2] V. Fuchs-Tarlovsky, Role of antioxidants in cancer therapy, Nutrition 29 (1) (2013) $15-21$.

[3] E.G. Phimister, N.S. Chandel, D.A. Tuveson, The promise and perils of antioxidants for cancer patients, N. Engl. J. Med. 371 (2) (2014) 177-178.

[4] C.L. Barden, W.J. Bramlage, Accumulation of antioxidants in apple peel as related to preharvest factors and superficial scald susceptibility of the fruit, J. Am. Soc. Hortic. Sci. 119 (2) (1994) 264-269.

[5] B.L. Halvorsen, K. Holte, M.C. Myhrstad, I. Barikmo, E. Hvattum, S.F. Remberg, A.-B. Wold, K. Haffner, H. Baugerød, L.F. Andersen, A systematic screening of total antioxidants in dietary plants, J. Nutr. 132 (3) (2002) 461-471.

[6] K. Wolfe, X. Wu, R.H. Liu, Antioxidant activity of apple peels, J. Agric. Food Chem. 51 (3) (2003) 609-614.

[7] B. Łata, Apple peel antioxidant status in relation to genotype, storage type and time, Sci. Hortic. 117 (1) (2008) 45-52.

[8] B. Łata, Relationship between apple peel and the whole fruit antioxidant content: year and cultivar variation, J. Agric. Food Chem. 55 (3) (2007) 663-671.

[9] K.L. Wolfe, R.H. Liu, Apple peels as a value-added food ingredient, J. Agric. Food Chem. 51 (6) (2003) 1676-1683.

[10] G. Noctor, C.H. Foyer, Ascorbate and glutathione: keeping active oxygen under control, Annu. Rev. Plant Physiol. Plant Mol. Biol. 49 (1998) 249-279.

[11] M.V. Eberhardt, C.Y. Lee, R.H. Liu, Nutrition: antioxidant activity of fresh apples, Nature 405 (6789) (2000) 903-904.

[12] C. Guo, J. Yang, J. Wei, Y. Li, J. Xu, Y. Jiang, Antioxidant activities of peel, pulp and seed fractions of common fruits as determined by FRAP assay, Nutr. Res. 23 (12) (2003) 1719-1726.

[13] A. Matthes, M. Schmitz-Eiberger, Polyphenol content and antioxidant capacity of apple fruit: effect of cultivar and storage conditions, J. Appl. Bot. Food Qual. 82 (2) (2012) 152-157.

[14] L. Panzella, M. Petriccione, P. Rega, M. Scortichini, A. Napolitano, A reappraisal of traditional apple cultivars from southern Italy as a rich source of phenols with superior antioxidant activity, Food Chem. 140 (4) (2013) 672-679.

[15] Y.-Z. Fang, S. Yang, G. Wu, Free radicals, antioxidants, and nutrition, Nutrition 18 (10) (2002) 872-879.

[16] M. Kalinowska, A. Bielawska, H. Lewandowska-Siwkiewicz, W. Priebe, W. Lewandowski, Apples: Content of phenolic compounds vs. variety, part of apple and cultivation model, extraction of phenolic compounds, biological properties, Plant Physiol. Biochem. 84 (2014) 169-188.

[17] K.W. Lee, Y.J. Kim, D.O. Kim, H.J. Lee, C.Y. Lee, Major phenolics in apple and their contribution to the total antioxidant capacity, J. Agric. Food Chem. 51 (22) (2003) 6516-6520.

[18] F. Shahidi, Y. Zhong, Measurement of antioxidant activity, J. Funct. Foods 18 (2015) 757-781.

[19] J.B.L. Tan, Y.Y. Lim, Critical analysis of current methods for assessing the in vitro antioxidant and antibacterial activity of plant extracts, Food Chem. 172 (2015) 814-822.

[20] A.A. Karaçelik, M. Küçük, Z. İskefiyeli, S. Aydemir, S. De Smet, B. Miserez, P. Sandra Antioxidant components of Viburnum opulus L. determined by on-line HPLC-UVABTS radical scavenging and LC-UV-ESI-MS methods, Food Chem. 175 (2015) 106-114.

[21] V.V. Roshchina, Autofluorescence of plant secreting cells as a biosensor and bioindicator reaction, J. Fluoresc. 13 (5) (2003) 403-420.

[22] N. Seawan, W. Vichit, A. Thakam, N. Thitipramote, P. Chaiwut, P. Pintathong, N. Thitilertdech, Antioxidant capacities, phenolic, anthocyanin and proanthocyanidin contents of pigmented rice extracts obtained by microwave-assisted method, Suranaree, J. Sci. Technol. 21 (4) (2014).

[23] S.F. Hagen, K.A. Solhaug, G.B. Bengtsson, G.I.A. Borge, W. Bilger, Chlorophyll fluorescence as a tool for non-destructive estimation of anthocyanins and total flavonoids in apples, Postharvest Biol. Technol. 41 (2) (2006) 156-163.

[24] M.N. Merzlyak, A.E. Solovchenko, A.I. Smagin, A.A. Gitelson, Apple flavonols during fruit adaptation to solar radiation: spectral features and technique for non-destructive assessment, J. Plant Physiol. 162 (2) (2005) 151-160.

[25] D.L. Betemps, J.C. Fachinello, S.P. Galarça, N.M. Portela, D. Remorini, R. Massai, G. Agati, Non-destructive evaluation of ripening and quality traits in apples using multiparametric fluorescence sensor, J. Sci. Food Agric. 92 (9) (2012) 1855-1864.

[26] P. Tacchini, A. Lesch, A. Neequaye, G. Lagger, J. Liu, F. Cortés-Salazar, H.H. Girault, Electrochemical pseudo-titration of water-soluble antioxidants, Electroanalysis 25 (2013) 922-930.

[27] M.F. Barroso, N. de-los-Santos-Alvarez, C. Delerue-Matos, M.B.P.P. Oliveira, Towards a reliable technology for antioxidant capacity and oxidative damage evaluation: Electrochemical (bio)sensors, Biosens. Bioelectron. 30 (1) (2011) 1-12.

[28] H. Li, F. Zhao, L. Yue, S. Li, F. Xiao, Nonenzymatic electrochemical biosensor based on novel hydrophilic ferrocene-terminated hyperbranched polymer and its application in glucose detection, Electroanalysis 28 (5) (2016) 1003-1011.

[29] K.Z. Brainina, D.P. Varzakova, E.L. Gerasimova, A chronoamperometric method for determining total antioxidant activity, J. Anal. Chem. 67 (4) (2012) 364-369.

[30] R.D.Q. Ferreira, S.J. Greco, M. Delarmelina, K.C. Weber, Electrochemical quantification of the structure/antioxidant activity relationship of flavonoids, Electrochim. Acta 163 (2015) 161-166.

[31] I.F. Benzie, J. Strain, The ferric reducing ability of plasma (FRAP) as a measure of "antioxidant power": the FRAP assay, Anal. Biochem. 239 (1) (1996) 70-76.

[32] P. Sun, F.O. Laforge, M.V. Mirkin, Scanning electrochemical microscopy in the 21st century, Phys. Chem. Chem. Phys. 9 (7) (2007) 802-823.
[33] G. Wittstock, M. Burchardt, S.E. Pust, Y. Shen, C. Zhao, Scanning electrochemical microscopy for direct imaging of reaction rates, Angew. Chem. Int. Ed. 46 (10) (2007) 1584-1617.

[34] S. Amemiya, A.J. Bard, F.-R.F. Fan, M.V. Mirkin, P.R. Unwin, Scanning electrochemical microscopy, Annu. Rev. Anal. Chem. 1 (2008) 95-131.

[35] A.J. Wain, Scanning electrochemical microscopy for combinatorial screening applications: a mini-review, Electrochem. Commun. 46 (2014) 9-12.

[36] Y. Li, X. Ning, Q. Ma, D. Qin, X. Lu, Recent advances in electrochemistry by scanning electrochemical microscopy, Trends Anal. Chem. 80 (2016) 242-254.

[37] D. Mandler, Scanning Electrochemical Microscopy (SECM), Encyclopedia of Applied Electrochemistry, Springer 2014, pp. 1826-1836.

[38] C.G. Zoski, Review-Advances in Scanning Electrochemical Microscopy (SECM), J. Electrochem. Soc. 163 (4) (2016) H3088-H3100.

[39] Y. Shao, M.V. Mirkin, Probing ion transfer at the liquid/liquid interface by scanning electrochemical microscopy (SECM), J. Phys. Chem. B 102 (49) (1998) 9915-9921.

[40] C. Tan, J. Rodríguez-López, J.J. Parks, N.L. Ritzert, D.C. Ralph, H.D. Abruna, Reactivity of monolayer chemical vapor deposited graphene imperfections studied using scanning electrochemical microscopy, ACS Nano 6 (4) (2012) 3070-3079.

[41] T. Matsue, Bioimaging with micro/nanoelectrode systems, Anal. Sci. 29 (2) (2013) $171-179$.

[42] T.-E. Lin, F. Cortés-Salazar, A. Lesch, L. Qiao, A. Bondarenko, H.H. Girault, Multiple scanning electrochemical microscopy mapping of tyrosinase in micro-contact printed fruit samples on polyvinylidene fluoride membrane, Electrochim. Acta 179 (2015) 57-64

[43] A. Hengstenberg, C. Kranz, W. Schuhmann, Facilitated tip-positioning and applications of non-electrode tips in scanning electrochemical microscopy using a shear force based constant-distance mode, Chem.Eur. J. 6 (9) (2000) 1547-1554.

[44] B. Ballesteros Katemann, A. Schulte, W. Schuhmann, Constant-distance mode Scanning Electrochemical Microscopy (SECM) - part I: adaptation of a non-optical shearforce-based positioning mode for SECM tips, Chem. Eur. J. 9 (9) (2003) 2025-2033.

[45] M. Etienne, A. Schulte, W. Schuhmann, High resolution constant-distance mode alternating current scanning electrochemical microscopy (AC-SECM), Electrochem. Commun. 6 (3) (2004) 288-293.

[46] R.T. Kurulugama, D.O. Wipf, S.A. Takacs, S. Pongmayteegul, P.A. Garris, J.E. Baur, Scanning electrochemical microscopy of model neurons: constant distance imaging, Anal. Chem. 77 (4) (2005) 1111-1117.

[47] Y. Takahashi, A.I. Shevchuk, P. Novak, B. Babakinejad, J. Macpherson, P.R. Unwin, H. Shiku, J. Gorelik, D. Klenerman, Y.E. Korchev, Topographical and electrochemical nanoscale imaging of living cells using voltage-switching mode scanning electrochemical microscopy, Proc. Natl. Acad. Sci. U. S. A. 109 (29) (2012) 11540-11545.

[48] A. Lesch, P.-C. Chen, F. Roelfs, C. Dosche, D. Momotenko, F. Cortés-Salazar, H.H. Girault, G. Wittstock, Finger probe array for topography-tolerant scanning electrochemical microscopy of extended samples, Anal. Chem. 86 (1) (2013) 713-720.

[49] Y. Takahashi, A.I. Shevchuk, P. Novak, Y. Murakami, H. Shiku, Y.E. Korchev, T. Matsue, Simultaneous noncontact topography and electrochemical imaging by SECM/SICM featuring ion current feedback regulation, J. Am. Chem. Soc. 132 (29) (2010) 10118-10126.

[50] L. Danis, M.E. Snowden, U.M. Tefashe, C.N. Heinemann, J. Mauzeroll, Development of nano-disc electrodes for application as shear force sensitive electrochemical probes, Electrochim. Acta 136 (2014) 121-129.

[51] A. Eifert, B. Mizaikoff, C. Kranz, Advanced fabrication process for combined atomic force-scanning electrochemical microscopy (AFM-SECM) probes, Micron 68 (2015) 27-35

[52] T. Sun, Y. Yu, B.J. Zacher, M.V. Mirkin, Scanning electrochemical microscopy of individual catalytic nanoparticles, Angew. Chem. Int. Ed. 53 (51) (2014) 14120-14123.

[53] M. Kang, D. Momotenko, A. Page, D. Perry, P.R. Unwin, Frontiers in nanoscale electrochemical imaging: faster, multifunctional, and ultrasensitive, Langmuir 32 (32) (2016) 7993-8008.

[54] J. Kim, C. Renault, N. Nioradze, N. Arroyo-Currás, K.C. Leonard, A.J. Bard, Electrocatalytic activity of individual Pt nanoparticles studied by nanoscale scanning electrochemical microscopy, J. Am. Chem. Soc. 138 (27) (2016) 8560-8568.

[55] T.E. Lin, A. Bondarenko, A. Lesch, H. Pick, F. Cortés-Salazar, H.H. Girault, Monitoring tyrosinase expression in non-metastatic and metastatic melanoma tissues by scanning electrochemical microscopy, Angew. Chem. Int. Ed. 55 (11) (2016) 3813-3816.

[56] A. Lesch, B. Vaske, F. Meiners, D. Momotenko, F. Cortés-Salazar, H.H. Girault, G. Wittstock, Parallel imaging and template-free patterning of self-assembled monolayers with soft linear microelectrode arrays, Angew. Chem. Int. Ed. 51 (41) (2012) 10413-10416.

[57] S. Kuss, D. Trinh, J. Mauzeroll, High-speed scanning electrochemical microscopy method for substrate kinetic determination: application to live cell imaging in human cancer, Anal. Chem. 87 (16) (2015) 8102-8106.

[58] S. Rapino, R. Marcu, A. Bigi, A. Soldà, M. Marcaccio, F. Paolucci, P.G. Pelicci, M. Giorgio, Scanning electro-chemical microscopy reveals cancer cell redox state, Electrochim. Acta 179 (2015) 65-73.

[59] P. Verboven, A. Nemeth, M.K. Abera, E. Bongaers, D. Daelemans, P. Estrade, E. Herremans, M. Hertog, W. Saeys, E. Vanstreels, B. Verlinden, M. Leitner, B. Nicolaï, Optical coherence tomography visualizes microstructure of apple peel, Postharvest Biol. Technol. 78 (2013) 123-132.

[60] D. Post-Beittenmiller, Biochemistry and molecular biology of wax production in plants, Annu. Rev. Plant Physiol. Plant Mol. Biol. 47 (1) (1996) 405-430.

[61] Z. Ju, W.J. Bramlage, Phenolics and lipid-soluble antioxidants in fruit cuticle of apples and their antioxidant activities in model systems, Postharvest Biol. Technol. 16 (2) (1999) 107-118.

[62] F. Cortés-Salazar, M. Träuble, F. Li, J.-M. Busnel, A.-L. Gassner, M. Hojeij, G. Wittstock, H.H. Girault, Soft stylus probes for scanning electrochemical microscopy, Anal. Chem. 81 (16) (2009) 6889-6896. 
[63] F. Cortés-Salazar, D. Momotenko, A. Lesch, G. Wittstock, H.H. Girault, Soft microelectrode linear array for scanning electrochemical microscopy, Anal. Chem. 82 (24) (2010) 10037-10044.

[64] C. Nunes Kirchner, K.H. Hallmeier, R. Szargan, T. Raschke, C. Radehaus, G. Wittstock, Evaluation of thin film titanium nitride electrodes for electroanalytical applications, Electroanalysis 19 (10) (2007) 1023-1031.

[65] A. Lesch, D. Momotenko, F. Cortés-Salazar, F. Roelfs, H.H. Girault, G. Wittstock, Highthroughput scanning electrochemical microscopy brushing of strongly tilted and curved surfaces, Electrochim. Acta 110 (2013) 30-41.

[66] B. Lertanantawong, A.P. O'Mullane, J. Zhang, W. Surareungchai, M. Somasundrum, A.M. Bond, Investigation of mediated oxidation of ascorbic acid by ferrocenemethanol using large-amplitude Fourier transformed ac voltammetry under quasi-reversible electron-transfer conditions at an indium tin oxide electrode, Anal. Chem. 80 (17) (2008) 6515-6525.
[67] J.L.J. Bezuidenhout, P.J. Robbertse, C. Kaiser, Anatomical investigation of lenticel development and subsequent discolouration of 'Tommy Atkins' and 'Keitt' mango (Mangifera indica L.) fruit, J. Hortic. Sci. Biotechnol. 80 (1) (2005) 18-22.

[68] H. Rymbai, M. Srivastav, R.R. Sharma, S.K. Singh, Lenticels on mango fruit: Origin, development, discoloration and prevention of their discoloration, Sci. Hortic. 135 (2012) 164-170.

[69] A. Bondarenko, F. Cortés-Salazar, M. Gheorghiu, S. Gaspar, D. Momotenko, L. Stanica, A. Lesch, E. Gheorghiu, H.H. Girault, Electrochemical push-pull probe: from scanning electrochemical microscopy to multimodal altering of cell microenvironment, Anal. Chem. 87 (8) (2015) 4479-4486.

[70] D. Momotenko, F. Cortés-Salazar, A. Lesch, G. Wittstock, H.H. Girault, Microfluidic push-pull probe for scanning electrochemical microscopy, Anal. Chem. 83 (13) (2011) 5275-5282. 